
cubic B-spline collocation method $\bullet-\bullet$ Vol. XXI, No. 1 


\title{
Stability and convergence of the cubic B-spline collocation method for time-fractional advection-diffusion equation
}

\author{
Allahbakhsh YAZDANI CHERATI ${ }^{1}$, Mahmod BADR $^{1}$, and Zohre AZIMI ${ }^{1}$ \\ ${ }^{1}$ University of Mazandaran
}

July 20, 2020

\begin{abstract}
In this paper, the time-fractional advection-diffusion equation is solved by a cubic B-spline collocation method. For the fractional derivative we use the concept of the fractional derivative of Capato. Calculating of this numerical scheme is very simple. The presented numerical scheme is unconditional stable and highly accurate. We also obtain some error estimations with L2-norm and L[?]-norm.
\end{abstract}

\section{Introduction}

[nindent=0em,lines $=3] \mathrm{N}$ owadays finding the solution of the fractional differential equations is important since many phenomena in the real-world like fluid flow, fluid mechanics, electrochemistry, mathematical biology, plasma physics, solid-state physics and other areas of application modeling are described by fractional differential equations(Ciesielski \& Leszczynski, 2003; Metzler \& Klafter, 2000).

A type of partial differential equations (PDEs) with fractional derivatives is fractional advection-diffusion equations (ADEs)(Chen et al., 2019; Huang et al., 2019; Amirat \& Münch, 2019). The fractional advectiondiffusion equation of fractional order $\alpha(0<\alpha<1)$ is

where $t$ is time coordinate and $x$ is spatial coordinate where $a \leq x \leq b . f(x, t)$ is a sufficiently smooth function. The equation (??) describes the advection-diffusion process in a finite plate with thickness $(b-a)$. $\beta$ and $\gamma$ are the positive constants fractional advection-diffusion coefficient and

$$
\frac{\partial^{\alpha} u(x, t)}{\partial t^{\alpha}}=\frac{1}{\Gamma(1-\alpha)} \int_{0}^{t} \frac{\partial u}{\partial \tau}(x, \tau) \frac{d \tau}{(t-\tau)^{\alpha}},
$$

is the fractional derivative in the Caputo's sense(Badr et al., 2018; Kilbas et al., 2006; Podlubny, 1999). 
cubic B-spline collocation method • - • Vol. XXI, No. 1

In general, it is not easy to derive the analytical and exact solutions of the fractional differential equations and the numerical solution of fractional differential equations has the attention of many researchers. That's why we use numerical methods to solve this set of equations. In the past decades, several numerical methods have been applied to solve fractional differential equations. These methods include finite difference methods(Yuste, 2006; Odibat, 2009), Adomian decomposition methods(Momani \& Odibat, 2008; Momani \& Odibat, 2006), variational iteration methods(Wu \& Lee, 2010; Odibat \& Momani, 2006), and other methods. Now, we will describe the latest achievement of researchers in this field. Caglar(Caglar \& Caglar, 2006; Caglar \& Caglar, 2009) in 2006 used the B-splines to solve the boundary value problems. In 2008, Saka worked on the Quintic B-spline collocation method for the numerical solution of the RLW equation(Saka et al., 2008) and a numerical solution of the RLW equation by Galerkin method using quartic B-splines in (Saka \& Dağ, 2008). In 2010, Zheng et al examined the finite difference method for the fractional advection-diffusion equation(Zheng et al., 2010). Javed et al in (Javed et al., 2017) researched some solutions of fractional order partial differential equations using the Adomian decomposition method in 2017. In 2019 Singh et al, presented numerical solution of nonlinear reaction-advection-diffusion equation(Singh et al., 2019). In 2019 Shah et al in (Shah et al., 2019) worked on the numerical Adomian method to solve fractional partial differential equations. But another method that is effective and accurate is the collocation method. The collocation method with piecewise polynomial functions is developed as a method for solving two-point boundary value problems. We consider the cubic B-spline as a piecewise polynomial function. This is because of two useful features of B-splines. The B-spline is that the continuity conditions are inherent and compared with other piecewise polynomial interpolation function, the B-spline is the smoothest interpolation function moreover, has small local support properties(Gholamian \& Saberi-Nadjafi, 2018; Li, 2012; Mittal \& Jain, 2012).

Most recently, the quantic B-spline collocation method is applied to obtain the numerical solution of threeorder partial integrodifferential equations in(Zhang et al., 2013).

The present paper studied the collocation method with the cubic B-spline as a piecewise polynomial to find a numerical solution of the fractional advection-diffusion equation. The paper is organized as follows:

In the second part, we express the cubic B-spline function and by discretizing equation (??) and using the Capato fractional derivative concept to discrete the fractional part of the equation (??), we arrive at the proposed numerical scheme to obtain the numerical solution of the fractional advection-diffusion equation. In section 3, we prove that the cubic B-spline collocation scheme for solving the fractional advection-diffusion equation with conditions (??) and (??) is unconditionally stable. In the fourth section, we obtain some convergence rank of the proposed scheme using some lemmas and theorems and show that the application of the proposed scheme presented in this paper is to solve the fractional advection-diffusion equation and by giving numerical examples in section 5, we show that the numerical scheme presented is accurate and efficient and the results are compared with the exact solutions and this paper ends with a conclusion in section 6 .

\section{Description of the numerical method}

Assume $[a, b]$ be the solution domain and suppose $\Delta: a=x_{0}<x_{1}<\cdots<x_{N}=b$, be a uniform partition in the interval $[a, b]$ with $x_{m}=a+m h,(m=0,1,2, \cdots, N)$ for $h=\frac{b-a}{N}$.

The cubic B-spline function $Q_{m}(x)$ for $m=-1, \cdots, N+1$ in the uniform partition $\Delta$ can be defined as follows;

$$
Q_{m}(x)=\frac{1}{h^{3}}
$$


cubic B-spline collocation method • - • Vol. XXI, No. 1

$\begin{cases}\left(x-x_{m-2}\right)^{3}, & {\left[x_{m-2}, x_{m-1}\right],} \\ h^{3}+3 h^{2}\left(x-x_{m-1}\right)+3 h\left(x-x_{m-1}\right)^{2}-3\left(x-x_{m-1}\right)^{3}, & {\left[x_{m-1}, x_{m}\right],} \\ h^{3}+3 h^{2}\left(x_{m+1}-x\right)+3 h\left(x_{m+1}-x\right)^{2}-3\left(x_{m+1}-x\right)^{3}, & {\left[x_{m}, x_{m+1}\right],} \\ \left(x_{m+2}-x\right)^{3}, & {\left[x_{m+1}, x_{m+2}\right],} \\ 0, & \text { o.w. }\end{cases}$

(1)

It is obvious that the support of the B-spline $Q_{m}(x)$ and its derivative is $\left[x_{m-2}, x_{m+2}\right]$. In (Kadalbajoo \& Arora, 2009), it was shown that $\left\{Q_{m}(x)\right\}_{m=-1}^{N+1}$ construct a basis for the functions over the solution domain. Let $u(x, t)$ and $U(x, t)$ are the analytical and numerical solutions of the differential equation (??), respectively. Therefore, the numerical solution can be approximated as

$$
u(x, t) \simeq U(x, t)=\sum_{m=-1}^{N+1} \delta_{m}(t) Q_{m}(x),
$$

where $\delta_{m}(t)$ are unknown time-dependent parameters that should be computed from the initial and boundary conditions using the collocation method.

Using (1) and (2), the values of $U(x, t), U_{x}(x, t)$ and $U_{x x}(x, t)$ are determined at the nodal points in terms of the time dependent parameters $\delta_{m}$ as follows:

$\left\{\begin{array}{l}U\left(x_{m}, t\right)=\delta_{m-1}(t)+4 \delta_{m}(t)+\delta_{m+1}(t), \\ h U_{x}\left(x_{m}, t\right)=3\left(\delta_{m+1}(t)-\delta_{m-1}(t)\right), \\ h^{2} U_{x x}\left(x_{m}, t\right)=6\left(\delta_{m-1}(t)-2 \delta_{m}(t)+\delta_{m+1}(t)\right) .\end{array}\right.$

(3)

In this part of the paper, we describe a numerical approach to approximate the solution of advectiondiffusion equation by cubic B-spline basis functions based on the collocation method. Let

$$
0=t_{0}<t_{1}<\cdots<t_{n}=T
$$

is a uniform partition on $[0, T]$ which $t_{k}=k \Delta t,(k=0,1,2, \cdots, n)$ for time step sizes $\Delta t=\frac{T}{n}$. Similar to the first order derivative, we use the following approximation to the time fractional derivative in Caputo 
cubic B-spline collocation method • - • Vol. XXI, No. 1

sense (Liu et al., 2007), we have(Badr et al., 2018):

where $\Gamma(\alpha)$ is the well-known Gamma function and the coefficients $b_{j}$ with $b_{0}=1$ are as

$$
b_{j}=(j+1)^{1-\alpha}-j^{1-\alpha}, \quad j=0,1, \cdots, n,
$$

$R_{\Delta t}^{k+1}$ is the truncation error of the approximation (??) and

$$
R_{\Delta t}^{k+1} \leq C_{u}(\Delta t)^{2-\alpha}
$$

(4)

where $C_{u}$ is a constant that is only related to $u(\operatorname{Lin} \& \mathrm{Xu}, 2007)$.

Lemma 1 The following relations hold for coefficients $b_{j}$ (Liu et al., 2007),

(1) $b_{0}=1$

(2) $b_{j}>0, \quad j=0,1, \cdots, n$,

(3) $b_{j}>b_{j+1}, \quad j=0,1, \cdots, n-1$.

By applying the difference form of the time derivative in (??), the fractional advection-diffusion equation (??) can be written as

where $U_{m}^{k}=U\left(x_{m}, t_{k}\right)$ and $f_{m}^{k}=f\left(x_{m}, t_{k}\right)$. Then using the collocation method and substituting (3) in (??) for $m=0,1, \cdots, N$ and $k=0,1, \cdots, n-1$, leads to the following recurrence difference formula corresponding to the parameters $\delta_{m}^{k}$, 
cubic B-spline collocation method • - • Vol. XXI, No. 1

where $r=\frac{(\Delta t)^{-\alpha}}{\Gamma(2-\alpha)}$ and $\delta_{m}^{k}=\delta_{m}\left(t_{k}\right)$. Equation (??) can be converted into matrix form as following

$$
A \boldsymbol{\delta}^{k+1}=B\left(b_{k} \boldsymbol{\delta}^{0}+\sum_{j=0}^{k-1}\left(b_{j}-b_{j+1}\right) \boldsymbol{\delta}^{k-j}\right)+\boldsymbol{f}^{k+1}, \quad k=0,1, \cdots, n-1,
$$

where $\boldsymbol{\delta}^{k}=\left[\delta_{-1}^{k}, \delta_{0}^{k}, \delta_{1}^{k}, \cdots, \delta_{N}^{k}, \delta_{N+1}^{k}\right]^{T}$ is the vector of time dependent unknown parameters, $A$ and $B$ are the coefficients matrices and $\boldsymbol{f}^{k}=\left[f_{0}^{k}, \cdots, f_{N}^{k}\right]^{T}$. These two tridiagonal matrices are in $(N+1) \times(N+3)$ dimensions. Thus we have $(N+1)$ equations with $(N+3)$ unknown parameters $\delta^{k}$ for $k=0,1, \cdots, n$. To make the system (5) solvable, by using the boundary conditions (??), the unknown parameters $\delta_{-1}^{k}$ and $\delta_{N+1}^{k}$ may be eliminated from the system as follows;

For $m=0$ and $m=N$, by using the relations (??) and (3) we have

$\left\{\begin{array}{l}U\left(x_{0}=a, t\right)=\delta_{-1}(t)+4 \delta_{0}(t)+\delta_{1}(t)=0, \\ U\left(x_{N}=b, t\right)=\delta_{N-1}(t)+4 \delta_{N}(t)+\delta_{N+1}(t)=0 .\end{array}\right.$

Thus, for every $k$ :

$\left\{\begin{array}{l}\delta_{-1}^{k}=-4 \delta_{0}^{k}-\delta_{1}^{k} \\ \delta_{N+1}^{k}=-4 \delta_{N}^{k}-\delta_{N-1}^{k}\end{array}\right.$

(6)

Therefore the matrix equation (5) reads

$$
A \boldsymbol{\delta}^{k+1}=B\left(b_{k} \boldsymbol{\delta}^{0}+\sum_{j=0}^{k-1}\left(b_{j}-b_{j+1}\right) \boldsymbol{\delta}^{k-j}\right)+\boldsymbol{f}^{k+1}
$$

(7)

where the matrices $A$ and $B$ can be defined as 


$$
\begin{aligned}
& A=
\end{aligned}
$$

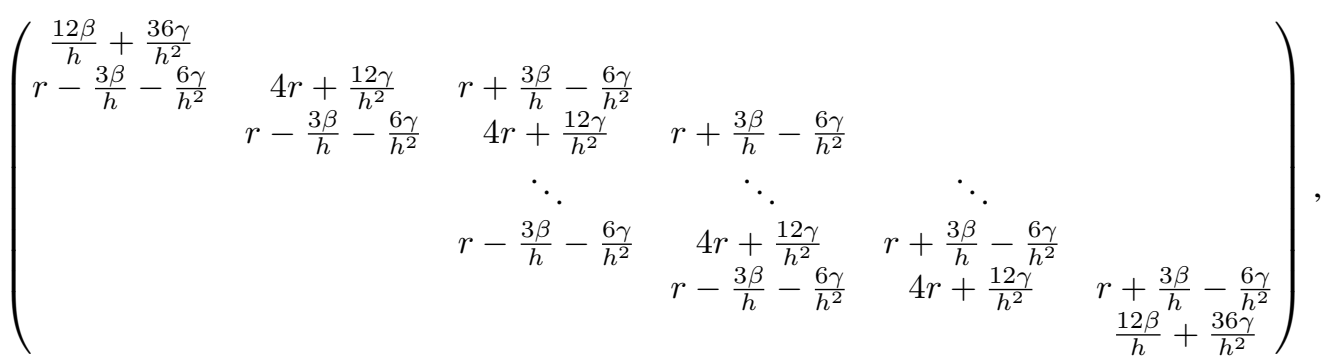

$$
\begin{aligned}
& B= \\
& \left(\begin{array}{cccccccc}
0 & 0 & 0 & 0 & & & & \\
r & 4 r & r & 0 & & & & \\
0 & r & 4 r & r & & & & \\
& & \ddots & \ddots & \ddots & & & \\
& & & & r & 4 r & r & 0 \\
& & & & 0 & r & 4 r & r \\
& & & & 0 & 0 & 0 & 0
\end{array}\right), \quad \boldsymbol{f}^{k+1}= \\
& \left(\begin{array}{c}
0 \\
\vdots \\
f^{k+1} \\
\vdots \\
0
\end{array}\right) .
\end{aligned}
$$

The matrix $A$ is a symmetric positive definite matrix in $(N+1) \times(N+1)$ order and therefore invertible. Having the initial vector $\delta^{0}$, the system (7) is solvable, has a unique solution and can be solved by an iterative algorithm such as Thomas algorithm. The starting vector $\boldsymbol{\delta}^{0}=\left[\delta_{-1}^{0}, \delta_{0}^{0}, \cdots, \delta_{N+1}^{0}\right]^{T}$ can be determined by (3) and initial conditions of the problem, as the following forms

$$
U\left(x_{m}, 0\right)=\delta_{m-1}^{0}+4 \delta_{m}^{0}+\delta_{m+1}^{0}=g\left(x_{m}\right), m=0,1, \cdots, N
$$

Therefore, the initial vector $\delta^{0}$ is determined from the following matrix equation; 
cubic B-spline collocation method $\bullet-\bullet$ Vol. XXI, No. 1

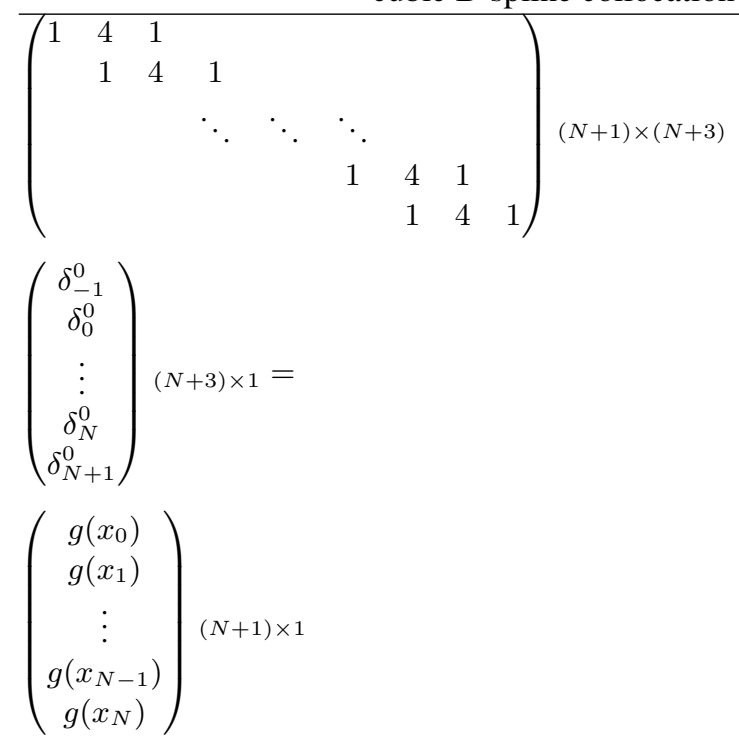

Corresponding to the time fractional derivative discretization, and as regards the matrix $A$ is positive definite, then the proposed numerical scheme (7) is consistent to the differential equation (??)-(??)(Rektorys \& Media, 2012).

\section{The stability of the method}

To investigate the stability conditions of the present numerical method, we use the Von-Neumann stability analysis and mathematical induction.

Theorem 1 The numerical scheme (??) for solving the initial and boundary value problem (??)-(??) is unconditionally stable.

Proof: We used the Von-Neumann processes and suppose that $f(x, t)=0$. Since the error of the method is only related to the time parameters $\delta_{m}^{k}$, denoting $\boldsymbol{e}^{k}:=\boldsymbol{\delta}^{k+1}-\boldsymbol{\delta}^{k}$ as the error of scheme at time level $k$, the numerical scheme (??) can be rewritten as

Then substituting the Fourier mode $e_{m}^{k}=v^{k} e^{i m \rho}(i:=\sqrt{-1})$ into (??) results 
cubic B-spline collocation method • - • Vol. XXI, No. 1

or

$$
v^{k+1}=Q\left\{b_{k} v^{0}+\sum_{s=0}^{k-1}\left(b_{s}-b_{s+1}\right) v^{k-s}\right\},
$$

where

$$
Q=\frac{2 r\left(1+2 \cos ^{2}\left(\frac{\rho}{2}\right)\right)}{2 r\left(1+2 \cos ^{2}\left(\frac{\rho}{2}\right)\right)+\frac{24 \gamma}{h^{2}} \sin ^{2}\left(\frac{\rho}{2}\right)+i \frac{6 \beta}{h} \sin (\rho)} .
$$

It is easy to verify that $|Q|^{2} \leq 1$. For $k=0$, (8) gives the following form: $v^{1}=Q b_{0} v^{0}$ and then

$$
\left|v^{1}\right|=|Q|\left|b_{0}\right|\left|v^{0}\right| \leq\left|v^{0}\right|
$$

Let $\left|v^{j}\right| \leq\left|v^{0}\right|, j=1,2, \ldots, k$. Hence, by equation (8) we have,

Thus, for every $k$, we have $\left|v^{k+1}\right| \leq\left|v^{0}\right|$. This relation shows that $\left|e^{k+1}\right| \leq\left|e^{0}\right|$, i.e., the error of this method in time level $k$, for every $k$, does not grow and is less than or equal to its initial error. So, the method is unconditionally stable.

\section{Error analysis}

In this section, we will give an error analysis of the present method and prove its convergence. To this end, we begin with some lemmas and theorems that give the properties of the B-spline functions and interpolation polynomials of spline type, which are important for the proof of uniform convergence of the numerical method. Assume $\Delta: a=x_{0}<x_{1}<\cdots<x_{N}=b$ be a partition od $[a, b]$. The spline functions $S_{k, \Delta}$ of degree $k$ are polynomial functions of degree $\mathrm{k}$, where $\Delta t=\frac{T}{n}$, that are $(k-1)$-times differentiable at the interior knots $x_{m}, 1 \leq m \leq N-1$, of $\Delta$. It can be seen that $S_{k, \Delta}$ is a vector space of dimension $N+k$ (Kadalbajoo \& Arora, 2009).

Theorem 2 The functions $\left\{Q_{-1}, Q_{0}, \ldots, Q_{N+1}\right\}$ form a basis of $S_{k, \Delta}$. 
cubic B-spline collocation method • - • Vol. XXI, No. 1

Proof: See (Kadalbajoo \& Arora, 2009).

Theorem 3 Let $s_{N} \in S_{k, \Delta}$ be a unique spline interpolating the solution $u(x, t)$ of initial boundary value problem (??)-(??). If $f(.,.) \in C^{2}[0,1]$ and $u(.,.) \in C^{4}[0,1]$, then there are constants $m_{i}$, independent of $h$ and $N$, so that for every $t \geqslant 0$, we have for $i=0,1,2$

$$
\left\|D^{i}\left(u(x, t)-s_{N}(x, t)\right)\right\|_{\infty} \leq m_{i} h^{4-i}
$$

where $D^{i}=\frac{\partial}{\partial x^{i}}$.

Proof: See (Kadalbajoo \& Arora, 2009).

Lemma 2 For the B-spline set $\left\{Q_{-1}, Q_{0}, \ldots, Q_{N+1}\right\}$, we have the following inequality

$$
\sum_{m=-1}^{N+1}\left|Q_{m}(x)\right| \leqslant 10, \quad a \leq x \leq b
$$

Proof: See (Kadalbajoo \& Arora, 2009).

The following theorem shows that the present method is convergence. It also gives an order to convergence of the numerical method in spatial coordinate.

Theorem 4 Let $U(x, t)$ be the collocation approximate from the space $S_{3, \Delta}$ to the solution $u(x, t)$ of the problem (??)-(??). This approximate exists and further, if $f(.,.) \in C^{2}[0,1]$, then there is a constant $C$ independent of $h$ that we have

$$
\|u(x, t)-U(x, t)\|_{\infty} \leqslant C h^{2}
$$

for every $t \geqslant 0$ and $h$ sufficiently small.

Proof: To prove of theorem, we use the best approximation of $u$ in $S_{k, \Delta}$ defined as $s_{N}(x, t)$ and write 
Thanks to theorem 3 , for $t \geqslant 0$, we have the following bound for the first term

$$
\left\|u(x, t)-s_{N}(x, t)\right\|_{\infty} \leqslant m_{0} h^{4}
$$

Corresponding to theorem 2 , the function $s_{N}(x, t)$ can be written in terms of B-spline basis as

$$
s_{N}(x, t)=\sum_{m=-1}^{N+1} \lambda_{m}(t) Q_{m}(x)
$$

Let $L s_{N}\left(x_{m}, t\right)=\hat{f}\left(x_{m}, t\right)$, for $m=0,1, \ldots, N$ in any time level $k$, in (??), we can write the difference $L\left(u\left(x_{m}, t\right)-s_{N}\left(x_{m}, t\right)\right)$ as follows

where $\eta_{m}^{k}=\delta_{m}^{k}-\lambda_{m}^{k}$ for $m=0, \ldots, N-1$ and $\zeta_{m}^{k}=h^{2}\left[f_{m}^{k}-\hat{f}_{m}^{k}\right]$ for $m=0,1, \ldots, N$. From inequality (9), it is evident that $\left|\zeta_{m}^{k}\right|=h^{2}\left|f_{m}^{k}-\hat{f}_{m}^{k}\right| \leq m_{0} h^{4}$. Let $\zeta^{k}=\max _{1 \leq m \leq N-1}\left|\zeta_{m}^{k}\right|, e_{m}^{k}=\left|\eta_{m}^{k}\right|$ and $e^{k}=\max _{1 \leq m \leq N-1}\left|e_{m}^{k}\right|$. From the initial condition of the problem, we have $e^{0}=0$ and setting $k=0$, we have

Using absolute values of $\zeta_{m}^{k}$ and $\eta_{m}^{k}$ with sufficiently small $h$ we have

$$
e_{m}^{1} \leq \frac{\zeta^{1}}{4 r h^{2}+12 \gamma} \leq \frac{m_{0} h^{6}}{4 r h^{2}+12 \gamma} \leq C_{1} h^{4}, \quad m=1,2, \ldots, N-1
$$

where $C_{1}$ is a constant independent of $h$. Also for $m=0$ and $m=N$, we have $e_{0}^{1} \leq C_{1} h^{3}$ and $e_{N}^{1} \leq C_{1} h^{3}$ and the values $e_{-1}^{1}$ and $e_{N+1}^{1}$ can be bounded using the boundary conditions given by (6), as follows:

$$
e_{-1}^{1} \leq C_{1} h^{2}, \quad e_{N+1}^{1} \leq C_{1} h^{2}
$$


Therefore with a constant $C_{1}$ independent of $h$, we have $e^{1} \leq C_{1} h^{2}$. Then, we use the mathematical induction on $k$ to prove the theorem. To this end, we first write the following form of (??):

Suppose that $C=\max _{1 \leq l \leq k} C_{l}$. As before, we can obtain the estimates for

$$
e_{m}^{k+1} \leq C_{l} h^{2}, \quad m=-1,0, \cdots, N+1
$$

Then, applying all of these relations, it can be concluded that for every $k$, there is a constant $w$ independent of $h$, so that $e^{k+1} \leq w h^{2}$. The above inequality together with lemma 2 enable us to obtain a bound for $\left\|U(x, t)-s_{N}(x, t)\right\|_{\infty}$, and hence a bound $\|u(x, t)-U(x, t)\|_{\infty}$. In particular

$$
U(x, t)-s_{N}(x, t)=\sum_{m=-1}^{N+1}\left(\delta_{m}(t)-\lambda_{m}(t)\right) Q_{m}(x)
$$

and thus

$$
\left\|s_{N}(x, t)-U(x, t)\right\|_{\infty} \leq 10 w h^{2}
$$

By considering the above results with the first inequality (??), we have

$$
\|u(x, t)-U(x, t)\|_{\infty} \leq M h^{2},
$$

where $M$ is a constant independent of $h$ and therefore the proof is completed.

Theorem 5 The numerical scheme (??) for solving fractional initial and boundary value problem (??)-(??) is convergent. 
cubic B-spline collocation method • - • Vol. XXI, No. 1

Proof: Let $u(x, t)$ be the exact solution of problem (??)-(??) and $U(x, t)$ be the approximate solution of $u$ by using scheme (??). Then, theorem 4 with relation (4) show that there are constants $C_{1}$ and $C_{2}$ such that

$$
\|u(x, t)-U(x, t)\|_{\infty} \leq C_{1} h^{2}+C_{2}(\Delta t)^{2-\alpha}=O\left(h^{2}+(\Delta t)^{2-\alpha}\right) .
$$

Thus, the presented numerical scheme is convergent and this completes the proof.

\section{Numerical examples}

In this section, numerical results of the proposed scheme for solving advection-diffusion equation were obtained by using the cubic B-spline basis functions and collocation method. We show that the numerical solution obtained above agree with those established in these examples.

The accuracy of the numerical method is measured by both the $L_{2}$ error norm

$$
\left\|u^{\text {exact }}-u_{h}\right\|_{2}^{2} \simeq h \sum_{j=0}^{N}\left|\left(u^{\text {exact }}\right)_{j}-\left(u_{h}\right)_{j}\right|^{2}
$$

and the $L_{\infty}$ error norm

$$
\left\|u^{\text {exact }}-u_{h}\right\|_{\infty} \simeq \max _{j}\left|\left(u^{\text {exact }}\right)_{j}-\left(u_{h}\right)_{j}\right|
$$

Example 1 Consider the following fractional initial and boundary value problem

$$
\begin{cases}u_{t}^{(\alpha)}(x, t)+\beta u_{x}(x, t)=\gamma u_{x x}(x, t)+f(x, t), & 0<x<1, \quad t \geq 0, \quad 0<\alpha<1, \\ u(0, t)=0, u(1, t)=0 & t \geq 0, \\ u(x, 0)=0, & 0 \leq x \leq 1,\end{cases}
$$

Let $\gamma=\beta=1$ and

$$
u(x, t)=t^{2} x(1-x)
$$


cubic B-spline collocation method • - • Vol. XXI, No. 1

is the exact solution of the equation (11). So

$$
f(x, t)=\frac{2 t^{2-\alpha}}{\Gamma(3-\alpha)} x(1-x)+t^{2}(3-2 x) .
$$

Table 1: Comparison of the errors of approximate solutions in $L_{2}$-norm and $L_{\infty}$-norm and convergence rate when $h=0.001$ and $t=0.5$.

\begin{tabular}{|c|c|c|c|c|c|}
\hline$\alpha$ & $i$ & $\Delta t$ & $L_{2}$-norm & $L_{\infty}$-norm & Rate \\
\hline \multirow{3}{*}{$\alpha=0.05$} & 1 & 0.5 & $0.1080367 \times 10^{-3}$ & $0.1524078 \times 10^{-3}$ & - \\
& 2 & 0.25 & $0.3121606 \times 10^{-4}$ & $0.4404524 \times 10^{-4}$ & 1.79 \\
& 3 & 0.125 & $0.1059793 \times 10^{-4}$ & $0.1495987 \times 10^{-4}$ & 1.55 \\
& 4 & 0.0625 & $0.2880765 \times 10^{-5}$ & $0.4073438 \times 10^{-5}$ & 1.87 \\
\hline \multirow{4}{*}{$\alpha=0.5$} & 1 & 0.5 & $0.2088790 \times 10^{-2}$ & $0.2945819 \times 10^{-2}$ & - \\
& 2 & 0.25 & $0.8217371 \times 10^{-3}$ & $0.1159131 \times 10^{-2}$ & 1.34 \\
& 3 & 0.125 & $0.3074675 \times 10^{-3}$ & $0.4337398 \times 10^{-3}$ & 1.41 \\
& 4 & 0.0625 & $0.1145140 \times 10^{-3}$ & $0.1615462 \times 10^{-3}$ & 1.42 \\
\hline \multirow{3}{*}{$\alpha=0.95$} & 1 & 0.5 & $0.6818897 \times 10^{-2}$ & $0.9614983 \times 10^{-2}$ & - \\
& 2 & 0.25 & $0.3630189 \times 10^{-2}$ & $0.5120979 \times 10^{-2}$ & 0.90 \\
& 3 & 0.125 & $0.1832533 \times 10^{-2}$ & $0.2585692 \times 10^{-2}$ & 0.98 \\
& 4 & 0.0625 & $0.8996515 \times 10^{-3}$ & $0.1369528 \times 10^{-2}$ & 1.02 \\
\hline
\end{tabular}

Table 2: Comparison of the errors of approximate solutions in $L_{2}$-norm and $L_{\infty}$-norm and convergence rate when $\Delta t=0.001$ and $t=0.5$.

\begin{tabular}{|c|c|c|c|c|c|}
\hline$\alpha$ & $i$ & $h$ & $L_{2}$-norm & $L_{\infty}$-norm & Rate \\
\hline \multirow{4}{*}{$\alpha=0.05$} & 1 & 0.5 & $0.1660360 \times 10^{-2}$ & $0.2858375 \times 10^{-2}$ & - \\
& 2 & 0.25 & $0.5100537 \times 10^{-3}$ & $0.9653501 \times 10^{-3}$ & 1.70 \\
& 3 & 0.125 & $0.1410359 \times 10^{-3}$ & $0.2801856 \times 10^{-3}$ & 1.85 \\
& 4 & 0.0625 & $0.3706499 \times 10^{-4}$ & $0.7549026 \times 10^{-4}$ & 1.92 \\
\hline \multirow{4}{*}{$\alpha=0.5$} & 1 & 0.5 & $0.2076855 \times 10^{-2}$ & $0.3182867 \times 10^{-2}$ & - \\
& 2 & 0.25 & $0.5099334 \times 10^{-3}$ & $0.9529274 \times 10^{-3}$ & 2.02 \\
& 3 & 0.125 & $0.1350067 \times 10^{-3}$ & $0.2760782 \times 10^{-3}$ & 1.91 \\
& 4 & 0.0625 & $0.3511436 \times 10^{-3}$ & $0.7482045 \times 10^{-3}$ & 1.94 \\
\hline \multirow{3}{*}{$\alpha=0.95$} & 1 & 0.5 & $0.6818897 \times 10^{-2}$ & $0.9614983 \times 10^{-2}$ & - \\
& 2 & 0.25 & $0.3630189 \times 10^{-2}$ & $0.5120979 \times 10^{-2}$ & 0.90 \\
& 3 & 0.125 & $0.1832533 \times 10^{-2}$ & $0.2585692 \times 10^{-2}$ & 0.98 \\
& 4 & 0.0625 & $0.8996515 \times 10^{-3}$ & $0.1369528 \times 10^{-2}$ & 1.02 \\
\hline
\end{tabular}

In Table 1 and Table 2, we present the results of applying the numerical scheme (??) to equation (11) with different $\alpha, \Delta t$ and $h$. In example 1 , first we consider $h=0.001$ and $t=0.5$ and we compare the numerical solutions of scheme (??) with the exact solution, and we report the error in $L_{2}$-norm and $L_{\infty}$-norm in Table 1 as well as the order of convergence of the numerical scheme to $L_{2}$. Now, considering $\Delta t=0.001$ and $t=0.5$, we show the error for different $\alpha$ and $h$ from $L_{2}$-norm and $L_{\infty}$-norm in Table 2 . Given the table 1 and 2, it can be concluded that the numerical scheme is convergent, that is, when the $h$ and $\Delta t$ tend to zero, the numerical solution of numerical scheme tends to the exact solution and the order of convergence is proportional to $O\left(h^{2}+\Delta t^{2-\alpha}\right)$. The order of convergence obtained by applying the numerical scheme (??) in equation (11) indicates the efficiency and accuracy of the desired numerical scheme. Now, in Figure 1 and Figure 2, we present the numerical results of the proposed scheme (??) for the approximate solution of the problem (11) for example 1. 
cubic B-spline collocation method $\bullet-\bullet$ Vol. XXI, No. 1

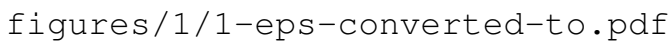

Figure 1: Numerical solutions of the equation (11) in example 1, when $\alpha=\frac{1}{4}$ 
cubic B-spline collocation method $\bullet-\bullet$ Vol. XXI, No. 1

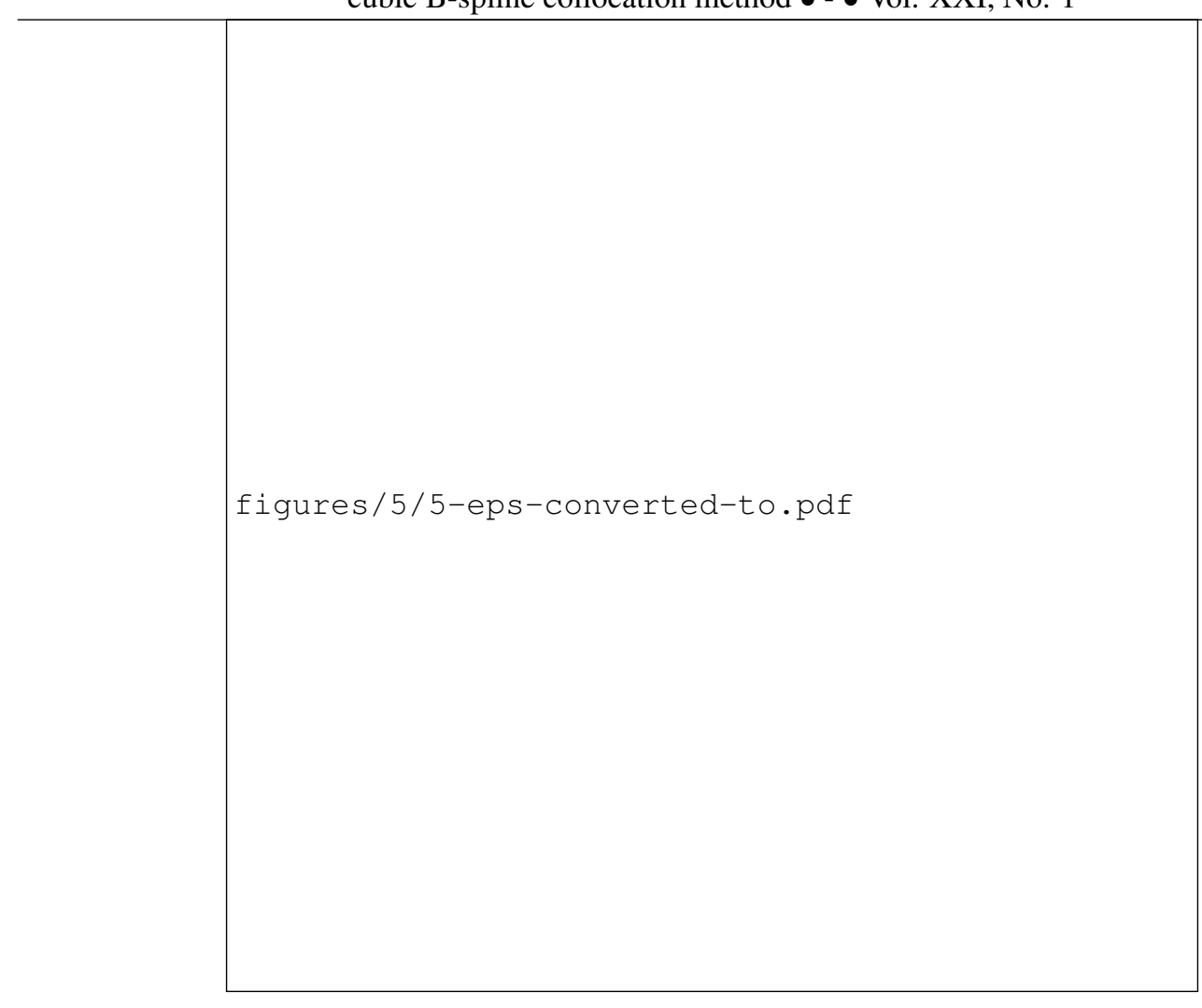

Figure 2: Numerical solutions of the equation (11) in example 1, when $\alpha=\frac{1}{3}$

Let $\alpha=\frac{1}{4}, n=40$ and $N=40$. In Figure 1, for different space $x=0.2$ and $x=0.4$, the results of applying the numerical scheme (??) in the equation of example 1 are shown. Now, we considering $n=40$, $N=40$ and $\alpha=\frac{3}{4}$. For different times in Figure 2, we comparison numerical solution of problem (11) with the exact solution. The computed results for the problem (11), corresponding to the time and spatial step size, show that the results show that the proposed numerical scheme is (??) accurate and efficient.

Example 2 As the second example, consider the nonhomogeneous advection-diffusion problem (11) with

and the exact solution is $u(x, t)=t \sin (2 \pi x)$. 
cubic B-spline collocation method $\bullet-\bullet$ Vol. XXI, No. 1

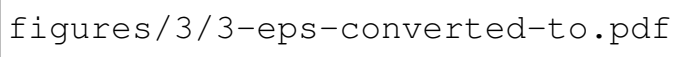

Figure 3: Numerical solutions of (11) in example 2, for $\alpha=\frac{3}{4}$. 
cubic B-spline collocation method • - • Vol. XXI, No. 1

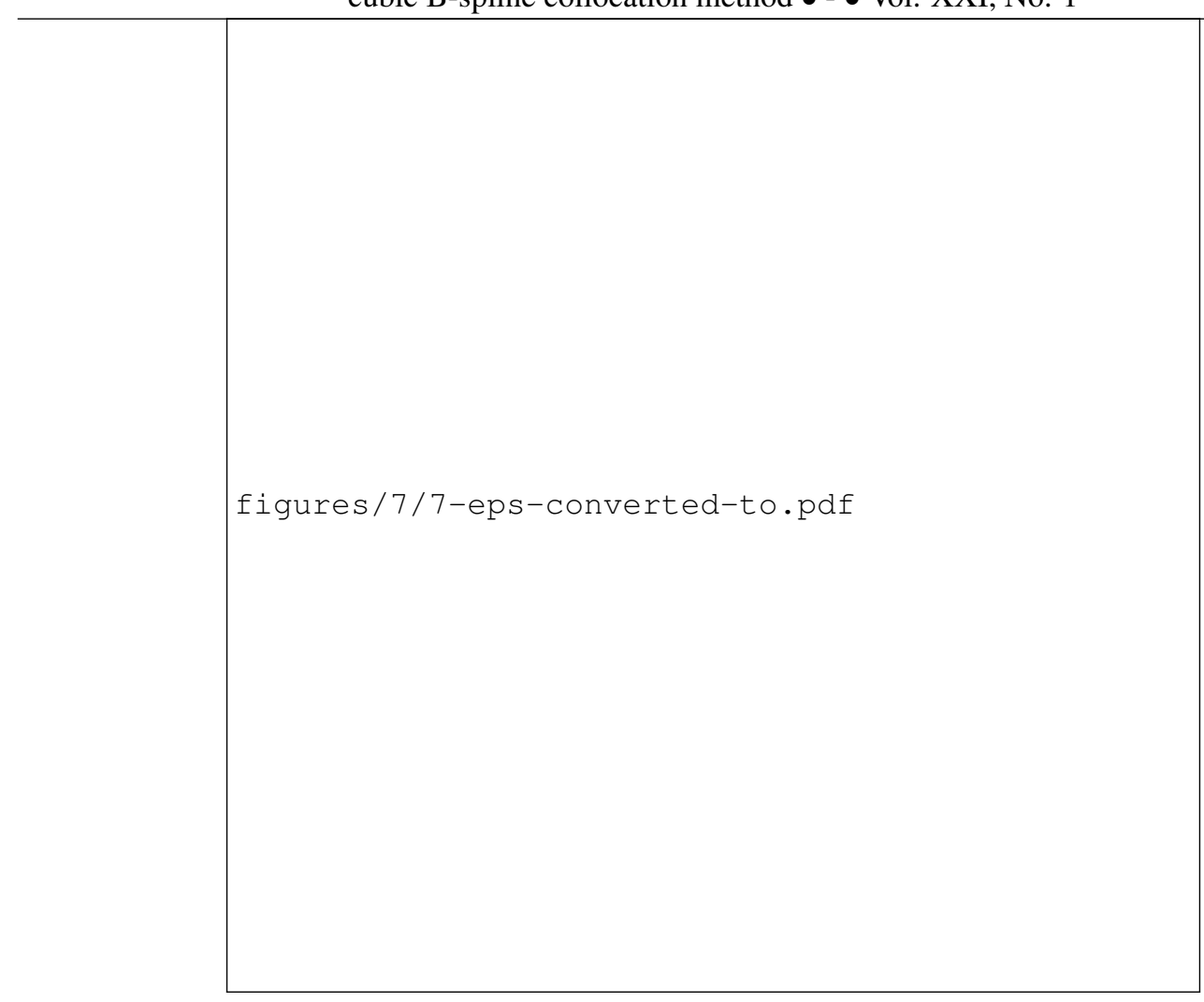

Figure 4: Numerical solutions of (11) in example 2, for $\alpha=\frac{2}{3}$.

Table 3: Comparison of errors of approximate solution for $\alpha=0.5$ in $t=0.5$.

\begin{tabular}{|c|c|c|c|c|c|}
\hline $\mathrm{i}$ & $\Delta t$ & $\mathrm{~h}$ & $L_{2}$-norm & $L_{\infty}$-norm & Order \\
\hline 1 & 0.25 & 0.25 & $0.4606681 \times 10^{-1}$ & $0.7442468 \times 10^{-1}$ & - \\
2 & 0.125 & 0.125 & $0.1086532 \times 10^{-1}$ & $0.1750665 \times 10^{-1}$ & 1.80 \\
3 & 0.0625 & 0.0625 & $0.2677718 \times 10^{-2}$ & $0.4392840 \times 10^{-2}$ & 2.02 \\
4 & 0.03125 & 0.03125 & $0.6670461 \times 10^{-3}$ & $0.1100802 \times 10^{-2}$ & 2.05 \\
5 & 0.015625 & 0.015625 & $0.1666378 \times 10^{-3}$ & $0.2751365 \times 10^{-3}$ & 2.03 \\
6 & 0.0078125 & 0.0078125 & $0.4170648 \times 10^{-4}$ & $0.6888954 \times 10^{-4}$ & 1.99 \\
\hline
\end{tabular}

The numerical errors at $\alpha=0.5$ and for several $h$ and $\Delta t$ have been shown in Table 3 and shows the error of the proposed method in comparison with the numerical and exact solutions. In Figure 3 and Figure 4 , the numerical solution of problem (11) are shown. The graphs of the numerical and analytical solutions are similar to each other.

\section{Conclusions}

In this study, a collocation method based on the B-spline basis functions method has been successfully used to obtain the numerical solutions of the time-fractional advection-diffusion equations. In this process, the time-fractional derivative is denoted in the Caputo sense and approximated by a backward difference formula. Finally, two examples were provided to demonstrate the applicability of the method. The results 
cubic B-spline collocation method • - • Vol. XXI, No. 1

which we obtained in this research show that the cubic B-spline collocation method is accurate and efficient for the numerical solution of time-fractional advection-diffusion equation.

\section{References}

Numerical Simulations of Anomalous Diffusion. Arxiv Preprint Math-Ph/030. (2003).

The Random Walk's Guide to Anomalous Diffusion: a Fractional Dynamics Approach. Physics Reports, 339 (1), 1-77. (2000).

Numerical Methods and Analysis for a Multi-Term Time-space Variable-Order Fractional Advection-diffusion Equations and Applications. Journal of Computational and Applied Mathematics, 352, 437452. (2019).

Carleman Estimates for the Time-Fractional Advection-Diffusion Equations and Applications. Inverse Problems, 35 (4), 04. (2019).

Asymptotic Analysis of an Advection-Diffusion Equation and Application to Boundary Controllability. Asymptotic Analysis, 112 (1-2), 59-106. (2019).

Stability of a Finite Volume Element Method for the Time-fractional Advection-diffusion Equation. Numerical Methods for Partial Differential Equations, 34 (5), 1459. (2018).

(2006).

Textit Fractional Differential Equations. Academic Press, San Diego. (1999).

Weighted Average Finite Difference Methods for Fractional Diffusion Equations. Journal of Computational Physics, 216 (1), 264-274. (2006).

Computational Algorithms for Computing the Fractional Derivatives of Functions. Mathematics and Computers in Simulation, 79 (7), 2013. (2009).

Numerical Solutions of the Space-time Fractional Advection-dispersion Equation. Numerical Methods for Partial Differential Equations: An International Journal, 24 (6), 1416. (2008).

Analytical Solution of a Time-Fractional Navier-stokes Equation by Adomian Decomposition Method. Applied Mathematics and Computation, 177 (2), 488-494. (2006).

Fractional Variational Iteration Method and Its Application. Physics Letters A, 374 (25), 2506. (2010).

Application of Variational Iteration Method to Nonlinear Differential Equations of Fractional Order. International Journal of Nonlinear Sciences and Numerical Simulation, 7 (1), 27-34. (2006).

(2006).

(2009).

Quintic B-Spline Collocation Method for Numerical Solution of the Rlw Equation. The Anziam Journal, 49 (3), 389-410. (2008).

(2008).

(2010).

Some Solutions of Fractional Order Partial Differential Equations Using Adomian Decomposition Method. Arxiv Preprint Arxiv:09207. (2017). 
cubic B-spline collocation method $\bullet-\bullet$ Vol. XXI, No. 1

Numerical Solution of Nonlinear Reaction-advection-diffusion Equation. Journal of Computational and Nonlinear Dynamics, 14 (4. (2019).

Application of Laplace-adomian Decomposition Method for the Analytical Solution of Third-Order Dispersive Fractional Partial Differential Equations. Entropy, 21 (4), 335. (2019).

Cubic B-Splines Collocation Method for a Class of Partial Integro-Differential Equation. Alexandria Engineering Journal, 57 (3), 2157. (2018).

Numerical Solution of Fractional Differential Equations Using Cubic B-Spline Wavelet Collocation Method. Communications in Nonlinear Science and Numerical Simulation, 17 (10), 3934. (2012).

Redefined Cubic B-Splines Collocation Method for Solving Convection-diffusion Equations. Applied Mathematical Modelling, 36 (11), 5555. (2012).

Quintic B-Spline Collocation Method for Fourth Order Partial Integro-Differential Equations with a Weakly Singular Kernel. Applied Mathematics and Computation, 219 (12), 6565. (2013).

(2009).

Stability and Convergence of the Difference Methods for the Space-time Fractional Advection-diffusion Equation. Applied Mathematics and Computation, 191 (1), 12-20. (2007).

Finite Difference/spectral Approximations for the Time-Fractional Diffusion Equation. Journal of Computational Physics, 225 (2), 1533. (2007).

(2012). 